\title{
A note on the almost sure central limit theorems for the maxima and sums
}

\section{Qing-pei Zang*}

\section{"Correspondence:}

zqphunhu@yahoo.com.cn

School of Mathematical Science,

Huaiyin Normal University, Huaian,

223300, China

\begin{abstract}
In this note, we derive, under some natural assumptions, a general pattern of the almost sure central limit theorem in the joint version for the maxima and sums.
\end{abstract}

Keywords: almost sure central limit theorem; maxima and partial sums; Lipschitz function

\section{Introduction and main results}

Let $\left\{X, X_{n} ; n \geq 1\right\}$ be a sequence of independent and identically distributed (i.i.d.) random variables and $S_{n}=\sum_{k=1}^{n} X_{k}, M_{n}=\max _{1 \leq k \leq n} X_{k}$ for $n \geq 1$. If $E(X)=0, E\left(X^{2}\right)=1$, then the classical almost sure central limit theorem (ASCLT) has the simplest form as follows:

$$
\lim _{n \rightarrow \infty} \frac{1}{\log n} \sum_{k=1}^{n} \frac{1}{k} I\left\{\frac{S_{k}}{\sqrt{k}} \leq x\right\}=\Phi(x) \quad \text { a.s. for all } x \in R,
$$

where, here and in the sequel, $I(A)$ is the indicator function of the event $A$ and $\Phi(x)$ stands for the standard normal distribution function. This result was firstly proved independently by Brosamler [1] and Schatte [2] under a stronger moment condition. Since then, this type of almost sure theorem, which mainly dealt with logarithmic average limit theorems, has been extended in various directions. In particular, Fahrner and Stadtmüller [3] and Cheng et al. [4] extended the almost sure convergence for partial sums to the case of maxima of i.i.d. random variables. Namely, under some natural conditions, they proved as follows:

$$
\lim _{n \rightarrow \infty} \frac{1}{\log n} \sum_{k=1}^{n} \frac{1}{k} I\left\{\frac{M_{k}-b_{k}}{a_{k}} \leq x\right\}=G(x) \quad \text { a.s. for all } x \in C_{G}
$$

where $C_{G}$ denotes the set of continuity points of $G$, and where $a_{k}>0$ and $b_{k} \in R$ satisfy

$$
\lim _{k \rightarrow \infty} P\left(\frac{M_{k}-b_{k}}{a_{k}} \leq x\right)=G(x) \quad \text { a.s. for any } x \in C_{G}
$$

with $G(x)$ being one of the extreme value distributions, i.e.,

$$
\begin{aligned}
& \wedge(x)=\exp \{-\exp (-x)\}, \\
& \Phi_{\alpha}(x)= \begin{cases}0, & x<0, \\
\exp \left\{-x^{-\alpha}\right\}, & x \geq 0,\end{cases}
\end{aligned}
$$

(0 2012 Zang; licensee Springer. This is an Open Access article distributed under the terms of the Creative Commons Attribution License (http://creativecommons.org/licenses/by/2.0), which permits unrestricted use, distribution, and reproduction in any medium, provided the original work is properly cited. 
for some $\alpha>0$, or

$$
\Psi_{\alpha}(x)= \begin{cases}\exp \left\{-(-x)^{\alpha}\right\}, & x<0 \\ 1, & x \geq 0\end{cases}
$$

for some $\alpha>0$. These three distributions are often called the Gumbel, the Frechet and the Weibull distributions, respectively.

For Gaussian sequences, Csáki and Gonchigdanzan [5] investigated, under some mild conditions, the validity of (1.2) for maxima of stationary Gaussian sequences. Furthermore, Chen and Lin [6] extended it to non-stationary Gaussian sequences. As for some other dependent random variables, Peligrad and Shao [7] and Dudziński [8] derived some corresponding results about ASCLT. In addition, the almost sure central limit theorem in the joint version for log-average of maxima and partial sums of independent and identically distributed random variables was obtained by Peng et al. [9], whereas the joint version of the almost sure limit theorem for log-average of maxima and partial sums of stationary Gaussian random variables was derived by Dudziński [10].

In statistical context, we are very concerned with the ASCLT in the joint version for the maxima and partial sums. The goal of this note is to investigate the general pattern of the ASCLT for the maxima and partial sums of i.i.d. random variables by the method provided by Hörmann [11]. He showed the following result.

Theorem A Let $X_{1}, X_{2}, \ldots$ be independent random variables with partial sums $S_{n}$. Assume that for some numerical sequences $a_{n}>0$ and $b_{n}$, we have

$$
\frac{S_{n}}{a_{n}}-b_{n} \stackrel{\mathcal{D}}{\rightarrow} H
$$

with some (possibly degenerate) distribution function $H$.

Suppose, moreover, that

$$
E\left|\frac{S_{n}}{a_{n}}-b_{n}\right|^{v}=O(1) \quad \text { for some } v>0
$$

and

$$
a_{k} / a_{l} \leq C(k / l)^{\beta} \quad(1 \leq k \leq l)
$$

for some positive constants $C, \beta$.

Assume finally that

$k d_{k} \ll 1$ and $d_{k} k^{\alpha}$ is nonincreasing for some $0<\alpha<1$,

and that

$$
d_{k}=O\left(\frac{D_{k}}{k\left(\log D_{k}\right)^{\rho}}\right) \quad \text { for some } \rho>0 \text {, where } D_{n}=\sum_{k=1}^{n} d_{k} \text {. }
$$


Then, iff is a bounded Lipschitz function on the real line or the indicator function of a Borel set $A \subset R$ with $\lambda(\partial A)=0$, we have

$$
\lim _{N \rightarrow \infty} \frac{1}{D_{N}} \sum_{k=1}^{N} d_{k} f\left(\frac{S_{k}}{a_{k}}-b_{k}\right)=\int_{-\infty}^{\infty} f(x) d H(x) \text { a.s. }
$$

Now, we may state our main result as follows.

Theorem 1.1 Let $\left\{X, X_{n} ; n \geq 1\right\}$ be a sequence of independent and identically distributed (i.i.d.) random variables with non-degenerate and continuous common distribution function $F$ satisfying $E(X)=0$ and $E\left(X^{2}\right)=1$. Suppose that, for a non-degenerate distribution $G$, there exist some numerical sequences $\left(a_{n}>0\right),\left(b_{n}\right)$ such that

$$
\frac{M_{n}-b_{n}}{a_{n}} \stackrel{\mathcal{D}}{\rightarrow} G
$$

Suppose, moreover, that the positive weights $d_{n}, n \geq 1$, satisfy the following conditions:

$\left(C_{1}\right) \liminf _{n \rightarrow \infty} n d_{n}>0$;

$\left(C_{2}\right) n^{\alpha} d_{n}$ is nonincreasing for some $0<\alpha<1$;

$\left(C_{3}\right) \lim \sup _{n \rightarrow \infty} n d_{n}\left(\log D_{n}\right)^{\rho} / D_{n}<\infty$ for some $\rho>0$, where $D_{n}=\sum_{k=1}^{n} d_{k}$.

Assume, in addition, that $f(x, y)$ is a bounded Lipschitz function. Then

$$
\lim _{n \rightarrow \infty} \frac{1}{D_{n}} \sum_{k=1}^{n} d_{k} f\left(\frac{S_{k}}{\sqrt{k}}, \frac{M_{k}-b_{k}}{a_{k}}\right)=\int_{-\infty}^{\infty} \int_{-\infty}^{\infty} f(x, y) \Phi(d x) G(d y) \quad \text { a.s. }
$$

Remark 1.2 Since a set of bounded Lipschitz functions is tight in a set of bounded continuous functions, Theorem 1.1 is true for all bounded continuous functions $f(x, y)$.

Remark 1.3 It can be seen by routine approximation arguments similar, e.g., to those in Lacey and Philipp [12] that, under the conditions of Theorem 1.1, the result in (1.4) holds for indicator functions, i.e.,

$$
\lim _{n \rightarrow \infty} \frac{1}{D_{n}} \sum_{k=1}^{n} d_{k} I\left(\frac{S_{k}}{\sqrt{k}} \leq x, \frac{M_{k}-b_{k}}{a_{k}} \leq y\right)=\Phi(x) G(y) \quad \text { a.s. }
$$

Remark 1.4 The result of Berkes and Csáki [13] shows that the a.s. central limit theorem remains valid even with the sequence of weights

$$
d_{k}=\frac{\exp \left((\log k)^{\alpha}\right)}{k} \quad \text { provided } \quad 0 \leq \alpha<\frac{1}{2}
$$

which at least includes a 'halfway' from logarithmic to ordinary averaging. Moreover, Hörmann [11] shows that this sequence obeys the a.s. central limit theorem for all $0 \leq \alpha<1$. Due to the similar conditions on the sequence of weights, our result also holds for this sequence provided $0 \leq \alpha<1$. 


\section{Proof of our main result}

The following notations will be used throughout this section: $S_{n}=\sum_{k=1}^{n} X_{k}, S_{k, n}=$ $\sum_{i=k+1}^{n} X_{i}, M_{n}=\max _{1 \leq i \leq n} X_{i}$, and $M_{k, n}=\max _{k+1 \leq i \leq n} X_{i}$ for $n \geq 1$. Furthermore, $a \ll b$ and $a \sim b$ stand for $a=O(b)$ and $\frac{a}{b} \rightarrow 1$, respectively, and $\Phi(x)$ is the standard normal distribution function. The proof of our main result is based on the following lemmas.

Lemma 1 Under the assumptions of Theorem 1.1, we have

$$
\operatorname{Cov}\left(f\left(\frac{S_{k}}{\sqrt{k}}, \frac{M_{k}-b_{k}}{a_{k}}\right), f\left(\frac{S_{l}}{\sqrt{l}}, \frac{M_{l}-b_{l}}{a_{l}}\right)\right) \ll\left(\frac{k}{l}\right)^{1 / 2}, \quad 1 \leq k \leq l
$$

Proof It is easy to see that

$$
\begin{aligned}
&\left|\operatorname{Cov}\left(f\left(\frac{S_{k}}{\sqrt{k}}, \frac{M_{k}-b_{k}}{a_{k}}\right), f\left(\frac{S_{l}}{\sqrt{l}}, \frac{M_{l}-b_{l}}{a_{l}}\right)\right)\right| \\
& \leq\left|\operatorname{Cov}\left(f\left(\frac{S_{k}}{\sqrt{k}}, \frac{M_{k}-b_{k}}{a_{k}}\right), f\left(\frac{S_{l}}{\sqrt{l}}, \frac{M_{l}-b_{l}}{a_{l}}\right)-f\left(\frac{S_{l}}{\sqrt{l}}, \frac{M_{k, l}-b_{l}}{a_{l}}\right)\right)\right| \\
&+\left|\operatorname{Cov}\left(f\left(\frac{S_{k}}{\sqrt{k}}, \frac{M_{k}-b_{k}}{a_{k}}\right), f\left(\frac{S_{l}}{\sqrt{l}}, \frac{M_{k, l}-b_{l}}{a_{l}}\right)-f\left(\frac{S_{k, l}}{\sqrt{l}}, \frac{M_{k, l}-b_{l}}{a_{l}}\right)\right)\right| \\
&+\left|\operatorname{Cov}\left(f\left(\frac{S_{k}}{\sqrt{k}}, \frac{M_{k}-b_{k}}{a_{k}}\right), f\left(\frac{S_{k, l}}{\sqrt{l}}, \frac{M_{k, l}-b_{l}}{a_{l}}\right)\right)\right| \\
&=: L_{1}+L_{2}+L_{3} .
\end{aligned}
$$

For $L_{3}$, we have, by the independence of $\left\{X_{n} ; n \geq 1\right\}$, that

$$
L_{3}=0 .
$$

Now, we are in a position to estimate $L_{1}$. From the fact that $f$ is bounded and Lipschitzian, it follows that

$$
\begin{aligned}
L_{1} & \ll E\left|f\left(\frac{S_{l}}{\sqrt{l}}, \frac{M_{l}-b_{l}}{a_{l}}\right)-f\left(\frac{S_{l}}{\sqrt{l}}, \frac{M_{k, l}-b_{l}}{a_{l}}\right)\right| \\
& \ll E\left(\min \left(\frac{M_{l}-M_{k, l}}{a_{l}}, 2\right)\right) \\
& =E\left(\min \left(\frac{M_{l}-M_{k, l}}{a_{l}}, 2\right)\right) I\left(M_{l} \neq M_{k, l}\right) \\
& \ll P\left(M_{l} \neq M_{k, l}\right) \\
& =P\left(M_{k}>M_{k, l}\right) \\
& =k \int_{-\infty}^{\infty}(F(x))^{l-k}(F(x))^{k-1} d F(x) \\
& \leq \int_{0}^{1} k t^{l-1} d t \\
& =\frac{k}{l}
\end{aligned}
$$


where we used the fact that

$$
\int_{-\infty}^{\infty} \psi(F(x)) d F(x) \leq \int_{0}^{1} \psi(t) d t
$$

for any nondecreasing function $\psi$ on $[0,1]$. In order to verify the last relation, let $F^{-1}(t)=$ $\sup \{x: F(x) \leq t\}$, and let $U$ be a random variable uniformly distributed on $(0,1)$. Then $F\left(F^{-1}(t)\right) \leq t$ for all $t \in(0,1)$ and the random variable $Y=F^{-1}(U)$ has distribution $F$. Thus, the left-hand side of the above inequality equals

$$
E \psi(F(Y))=E \psi\left(F\left(F^{-1}(U)\right)\right) \leq E \psi(U)=\int_{0}^{1} \psi(t) d t
$$

as claimed.

By the fact that $f$ is Lipschitzian and due to the Cauchy-Schwarz inequality, we have

$$
\begin{aligned}
L_{2} & \ll E\left|f\left(\frac{S_{l}}{\sqrt{l}}, \frac{M_{k, l}-b_{l}}{a_{l}}\right)-f\left(\frac{S_{k, l}}{\sqrt{l}}, \frac{M_{k, l}-b_{l}}{a_{l}}\right)\right| \\
& \ll E\left|\frac{S_{k}}{\sqrt{l}}\right| \leq \frac{1}{\sqrt{l}}\left(E S_{k}^{2}\right)^{\frac{1}{2}} \\
& =\left(\frac{k}{l}\right)^{\frac{1}{2}} .
\end{aligned}
$$

Thus, using (2.1)-(2.3), we get the desired result.

Let $f$ and $\left\{X, X_{n} ; n \geq 1\right\}$ be such as in the statement of Theorem 1.1 and

$$
\begin{aligned}
& \xi_{k}=f\left(\frac{S_{k}}{\sqrt{k}}, \frac{M_{k}-b_{k}}{a_{k}}\right)-E f\left(\frac{S_{k}}{\sqrt{k}}, \frac{M_{k}-b_{k}}{a_{k}}\right), \\
& \xi_{k, l}=f\left(\frac{S_{k, l}}{\sqrt{l}}, \frac{M_{k, l}-b_{l}}{a_{l}}\right)-E f\left(\frac{S_{k, l}}{\sqrt{l}}, \frac{M_{k, l}-b_{l}}{a_{l}}\right) .
\end{aligned}
$$

We will also prove the following auxiliary result.

Lemma 2 Let $p$ be a positive integer. Then for $1 \leq k \leq l$, we have

$$
E\left|\xi_{l}-\xi_{k, l}\right|^{p} \ll\left(\frac{k}{l}\right)^{1 / 2}
$$

Proof Without loss of generality, we may assume that $|f| \leq 1$. Thus, we have

$$
E\left|\xi_{l}-\xi_{k, l}\right|^{p} \leq 4^{p-1} E\left|\xi_{l}-\xi_{k, l}\right|
$$

Furthermore, we obtain that

$$
\begin{aligned}
E\left|\xi_{l}-\xi_{k, l}\right| & \leq 2 E\left|f\left(\frac{S_{l}}{\sqrt{l}}, \frac{M_{l}-b_{l}}{a_{l}}\right)-f\left(\frac{S_{k, l}}{\sqrt{l}}, \frac{M_{k, l}-b_{l}}{a_{l}}\right)\right| \\
& \leq 2 E\left|f\left(\frac{S_{k, l}}{\sqrt{l}}, \frac{M_{k, l}-b_{l}}{a_{l}}\right)-f\left(\frac{S_{l}}{\sqrt{l}}, \frac{M_{k, l}-b_{l}}{a_{l}}\right)\right|
\end{aligned}
$$




$$
\begin{aligned}
& +2 E\left|f\left(\frac{S_{l}}{\sqrt{l}}, \frac{M_{l}-b_{l}}{a_{l}}\right)-f\left(\frac{S_{l}}{\sqrt{l}}, \frac{M_{k, l}-b_{l}}{a_{l}}\right)\right| \\
\ll & P\left(M_{l} \neq M_{k, l}\right)+E\left|\frac{S_{k}}{\sqrt{l}}\right| \\
\ll & \frac{k}{l}+\frac{1}{\sqrt{l}}\left(E S_{k}^{2}\right)^{1 / 2} \\
\ll & \left(\frac{k}{l}\right)^{1 / 2} .
\end{aligned}
$$

The relations in (2.4), (2.5) imply the claim in Lemma 2.

The following lemma will also be used.

Lemma 3 Let $p$ be a positive integer. Then for $1 \leq k \leq m \leq n$, we have

$$
E\left|\sum_{l=m}^{n} d_{l}\left(\xi_{l}-\xi_{k, l}\right)\right|^{p} \ll\left(\sum_{l=m}^{n} l d_{l}^{2}\right)^{\frac{p}{2}}
$$

Proof We can write

$$
\left(\sum_{l=m}^{n} d_{l}\left(\xi_{k}-\xi_{k, l}\right)\right)^{p}=\sum_{l_{1}=m}^{n} \cdots \sum_{l_{p}=m}^{n} d_{l_{1}} \cdots d_{l_{p}}\left(\xi_{l_{1}}-\xi_{k, l_{1}}\right) \cdots\left(\xi_{l_{p}}-\xi_{k, l_{p}}\right) .
$$

Thus, using the Hölder inequality, the Cauchy-Schwarz inequality and Lemma 2, we derive

$$
\begin{aligned}
E\left|\sum_{l=m}^{n} d_{l}\left(\xi_{l}-\xi_{k, l}\right)\right|^{p} & \leq \sum_{l_{1}=m}^{n} \cdots \sum_{l_{p}=m}^{n} d_{l_{1}} \cdots d_{l_{p}}\left(E\left|\xi_{l_{1}}-\xi_{k, l_{1}}\right|^{p} \cdots E\left|\xi_{l_{p}}-\xi_{k, l_{p}}\right|^{p}\right)^{\frac{1}{p}} \\
& \ll \sqrt{k} \sum_{l_{1}=m}^{n} \cdots \sum_{l_{p}=m}^{n} d_{l_{1}} \cdots d_{l_{p}} l_{1}^{-\frac{1}{2 p}} \cdots l_{p}^{-\frac{1}{2 p}} \\
& =\sqrt{k}\left(\sum_{l=m}^{n} d_{l} l^{\frac{1}{2 p}}\right)^{p} \\
& \leq \sqrt{m}\left(\sum_{l=m}^{n} l d_{l}^{2}\right)^{\frac{p}{2}}\left(\sum_{l=m}^{n} l^{-\frac{1}{p}-1}\right)^{\frac{p}{2}} .
\end{aligned}
$$

This and the relation

$$
\sum_{l=m}^{n} l^{-\frac{1}{p}-1} \leq p \frac{1}{(m-1)^{\frac{1}{p}}} \ll m^{-\frac{1}{p}}
$$

imply the desired result.

We will also prove the following lemma. 
Lemma 4 For every $p \in \mathbb{N}$, we have

$$
E\left|\sum_{k=1}^{n} d_{k} \xi_{k}\right|^{p} \ll\left(\sum_{1 \leq k \leq l \leq n} d_{k} d_{l}\left(\frac{k}{l}\right)^{1 / 2}\right)^{\frac{p}{2}}
$$

Proof This lemma can be obtained from Lemmas 1 and 3 by making slight changes in the proof of Lemma 4 of Hörmann [11].

The following result will be needed in the proof of our main result.

Lemma 5 Suppose that $\eta<\rho$, where $\rho$ satisfies the assumption in $\left(C_{3}\right)$ of Theorem 1.1. We have

$$
\sum_{1 \leq k \leq l \leq n} d_{k} d_{l}\left(\frac{k}{l}\right)^{1 / 2}=O\left(\frac{D_{n}^{2}}{\left(\log D_{n}\right)^{\eta}}\right), \quad \text { where } D_{n}=\sum_{i=1}^{n} d_{i}
$$

Proof This result follows from Lemma 5 in Hörmann [11].

Proof of Theorem 1.1 Firstly, by Theorem 1.1. in Hsing [14] and our assumptions, we have

$$
\lim _{n \rightarrow \infty} P\left(\frac{S_{n}}{\sqrt{n}} \leq x, \frac{M_{n}-b_{n}}{a_{n}} \leq y\right)=\Phi(x) G(y) \quad \text { for } x, y \in R
$$

Then, in view of the dominated convergence theorem, we have

$$
E f\left(\frac{S_{n}}{\sqrt{n}}, \frac{M_{n}-b_{n}}{a_{n}}\right) \rightarrow \int_{-\infty}^{\infty} \int_{-\infty}^{\infty} f(x, y) \Phi(d x) G(d y) .
$$

Hence, in order to complete the proof, it is sufficient to show

$$
\lim _{n \rightarrow \infty} \frac{1}{D_{n}} \sum_{k=1}^{n} d_{k}\left(f\left(\frac{S_{k}}{\sqrt{k}}, \frac{M_{k}-b_{k}}{a_{k}}\right)-E f\left(\frac{S_{k}}{\sqrt{k}}, \frac{M_{k}-b_{k}}{a_{k}}\right)\right)=0 \quad \text { a.s. }
$$

This follows from Lemmas 4 and 5 by applying similar arguments to those used in Hörmann [11].

In fact, from Lemmas 4 and 5 and the Markov inequality, we derive

$$
P\left(\left|\sum_{k=1}^{n} d_{k} \xi_{k}\right|>\varepsilon D_{n}\right) \ll\left(\log D_{n}\right)^{\frac{-p \eta}{2}} \quad \text { for any } \varepsilon, p \in N \text { and large enough } n \text {. }
$$

By $\left(C_{3}\right)$, we have $\frac{D_{n_{j}+1}}{D_{n_{j}}} \rightarrow 1$. Thus, we can choose an increasing subsequence $\left(n_{j}\right)$ such that $D_{n_{j}}=O\left(\exp \left(j^{\frac{1}{2}}\right)\right)$. Then, choosing $p>\frac{4}{\eta}$ and using Borel-Cantelli lemma, we derive

$$
\frac{1}{D_{n_{j}}} \sum_{i=1}^{n_{j}} d_{i} \xi_{i} \rightarrow 0 \quad \text { a.s. }
$$


For $n_{j} \leq n<n_{j+1}$, we have

$$
\frac{1}{D_{n}}\left|\sum_{k=1}^{n} d_{k} \xi_{k}\right| \leq \frac{1}{D_{n_{j}}}\left|\sum_{i=1}^{n_{j}} d_{i} \xi_{i}\right|+2\left(\frac{D_{n_{j+1}}}{D_{n_{j}}}-1\right) \text { a.s. }
$$

Since $\frac{D_{n_{j+1}}}{D_{n_{j}}} \rightarrow 1$, the convergence of the subsequence implies that the whole sequence converges almost surely. This completes the proof of Theorem 1.1.

\section{Competing interests}

The authors declare that they have no competing interests.

\section{Acknowledgements}

The author would like to thank the Editor and the two referees for careful reading of the paper and for their comments which have led to the improvement of this work. This work was supported by the Natural Science Research Project of Ordinary Universities in Jiangsu Province, P.R. China. Grant No. 12KJB110003.

\section{Received: 10 April 2012 Accepted: 4 September 2012 Published: 8 October 2012}

\section{References}

1. Brosamler, GA: An almost everywhere central limit theorem. Math. Proc. Camb. Philos. Soc. 104, 561-574 (1988)

2. Schatte, P: On strong versions of the central limit theorem. Math. Nachr. 137, 249-256 (1988)

3. Fahrner, I, Stadtmüller, U: On almost sure max-limit theorems. Stat. Probab. Lett. 37, 229-236 (1998)

4. Cheng, SH, Peng, L, Qi, YC: Almost sure convergence in extreme value theory. Math. Nachr. 190, 43-50 (1998)

5. Csáki, E, Gonchigdanzan, K: Almost sure limit theorems for the maximum of stationary Gaussian sequences. Stat. Probab. Lett. 58, 195-203 (2002)

6. Chen, SQ, Lin, ZY: Almost sure max-limits for nonstationary Gaussian sequence. Stat. Probab. Lett. 76, 1175-1184 (2006)

7. Peligrad, M, Shao, QM: A note on the almost sure central limit theorem for weakly dependent random variables. Stat. Probab. Lett. 22, 131-136 (1995)

8. Dudziński, M: A note on the almost sure central limit theorem for some dependent random variables. Stat. Probab. Lett. 61, 31-40 (2003)

9. Peng, ZX, Wang, LL, Nadarajah, S: Almost sure central limit theorem for partial sums and maxima. Math. Nachr. 282 632-636 (2009)

10. Dudziński, M: The almost sure central limit theorems in the joint version for the maxima and sums of certain stationary Gaussian sequences. Stat. Probab. Lett. 78, 347-357 (2008)

11. Hörmann, S: An extension of almost sure central limit theory. Stat. Probab. Lett. 76, 191-202 (2006)

12. Lacey, MT, Philipp, W: A note on the almost sure central limit theorem. Stat. Probab. Lett. 9, 201-205 (1990)

13. Berkes, I, Csáki, E: A universal result in almost sure central limit theory. Stoch. Process. Appl. 94, 105-134 (2001)

14. Hsing, T: A note on the asymptotic independence of the sum and maximum of strongly mixing stationary random variables. Ann. Probab. 23, 938-947 (1995)

\section{Submit your manuscript to a SpringerOpen ${ }^{\circ}$ journal and benefit from:}

- Convenient online submission

- Rigorous peer review

- Immediate publication on acceptance

- Open access: articles freely available online

- High visibility within the field

- Retaining the copyright to your article 